\title{
Proliferative verrucous leukoplakia, squamous cell carcinoma and axillary metastasis
}

\author{
Adriele-Ferreira Gouvêa ${ }^{1}$, Adriana-Elisa-Brasil Moreira ${ }^{2}$, Rodrigo-Ribas-Dias Reis ${ }^{2}$, Oslei-Paes de Almeida ${ }^{1}$, \\ Márcio-Ajudarte Lopes ${ }^{1}$
}

${ }^{1}$ DDS, MSC, DDS, PHD Department of Oral Diagnosis-Oral Pathology Section, Piracicaba Dental School-University of Campinas, Piracicaba - São Paulo State, Brazil

${ }^{2}$ MD Private office and Santa Casa Hospital, Piracicaba, São Paulo, Brazil

Correspondence:

Departamento de Diagnóstico Oral

Faculdade de Odontologia de Piracicaba-UNICAMP

Av. Limeira, 901- Areião

Caixa Postal 52, CEP: 13414-903

Piracicaba-SP, Brazil

adrielefg@yahoo.com.br

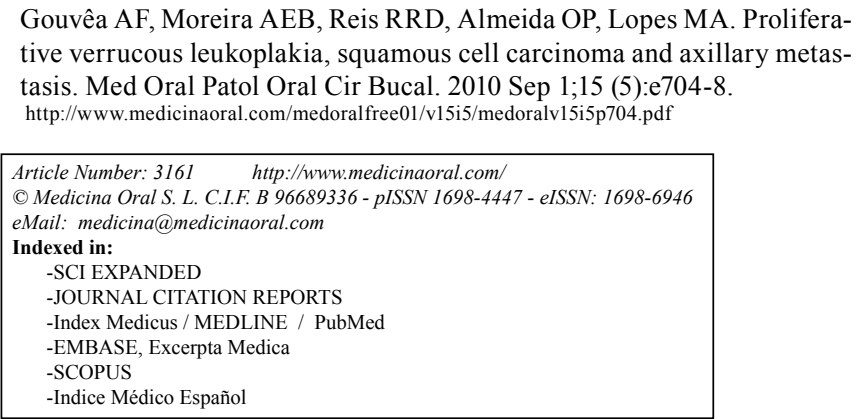

\begin{abstract}
Proliferative verrucous leukoplakia (PVL) is an aggressive form of oral leukoplakia with multifocal presentation, high rates of recurrence and malignant transformation. Although development of regional lymph node metastasis is relatively frequent in patients with oral squamous cell carcinoma, axillary metastasis is quite uncommon. This paper presents a case of a 64-year-old female patient who was diagnosed with PVL and developed five oral squamous cell carcinomas and later an axillary lymph node metastasis.
\end{abstract}

Key words: Proliferative verrucous leukoplakia, squamous cell carcinoma, axillary metastasis.

\section{Introduction}

Proliferative verrucous leukoplakia (PVL) was first described by Hansen et al., in 1985 (1) as a distinct and aggressive type of oral leukoplakia with unknown etiology affecting more commonly women in the sixth decade (female to male ratio $=4: 1$ ). PVL presents multifocal or diffuse extension, tendency to recur after treatment and high risk of malignant transformation (1-8). Several studies have reported high rates of malignant transformation which varies from $60 \%$ to $100 \%(2,3,9,10)$.

Metastasis from oral squamous cell carcinoma (SCC) follows a predictable way of lymphatic drainage that varies particularly according to the anatomic site, size and histopathological features (11). Eventually, metastasis can follow an unpredictable pattern and involve unexpected lymph nodes such as contralateral cervical lymph nodes, low jugular-carotid lymph nodal chain and even axillary lymph nodes $(12,13)$.

There are few reports published in the English-language literature about axillary metastasis from head and neck squamous cell carcinoma (HNSCC) and fewer from oral SCC (14). This paper presents the case of a 64 -yearold female patient who was diagnosed with PVL and developed five oral SCC and later an axillary lymph node 
metastasis. To the best of our knowledge this is the first case report of axillary metastasis in a patient with oral SCC that developed from PVL.

\section{Case Report}

A 64-year-old Caucasian woman, non-smoker, nondrinker, was referred to the Orocentro (Oral Diagnosis Clinic-Piracicaba Dental School-UNICAMP, Sao Paulo, Brazil) complaining of a tongue wound. She reported the onset of a white lesion on the tongue, four years before, which at that time was biopsied and showed extensive epithelial acanthosis and inflammatory infiltrate in the connective tissue. On intra-oral examination it was observed a very extensive leukoerythroplastic le- sion with some verrucous and ulcerated areas on the dorsum and ventral surface of the tongue (Fig. 1A). An incisional biopsy was performed and a well differentiated superficially invasive SCC was diagnosed (Table 1). A body screening was performed (nasofibrolaryngoscopy and chest X-ray) and no other neoplasia was detected. A left hemiglossectomy and left supraomohyoid neck dissection were performed, which showed no lymph node metastases. Because of the tumor size, the patient received post-operative radiotherapy to the left neck at a dose of 60Gy. Almost six months later, she presented leukoplastic lesions on the soft palate, tip and ventral surface of the tongue, right and left lateral border of the tongue, right buccal mucosa, right upper
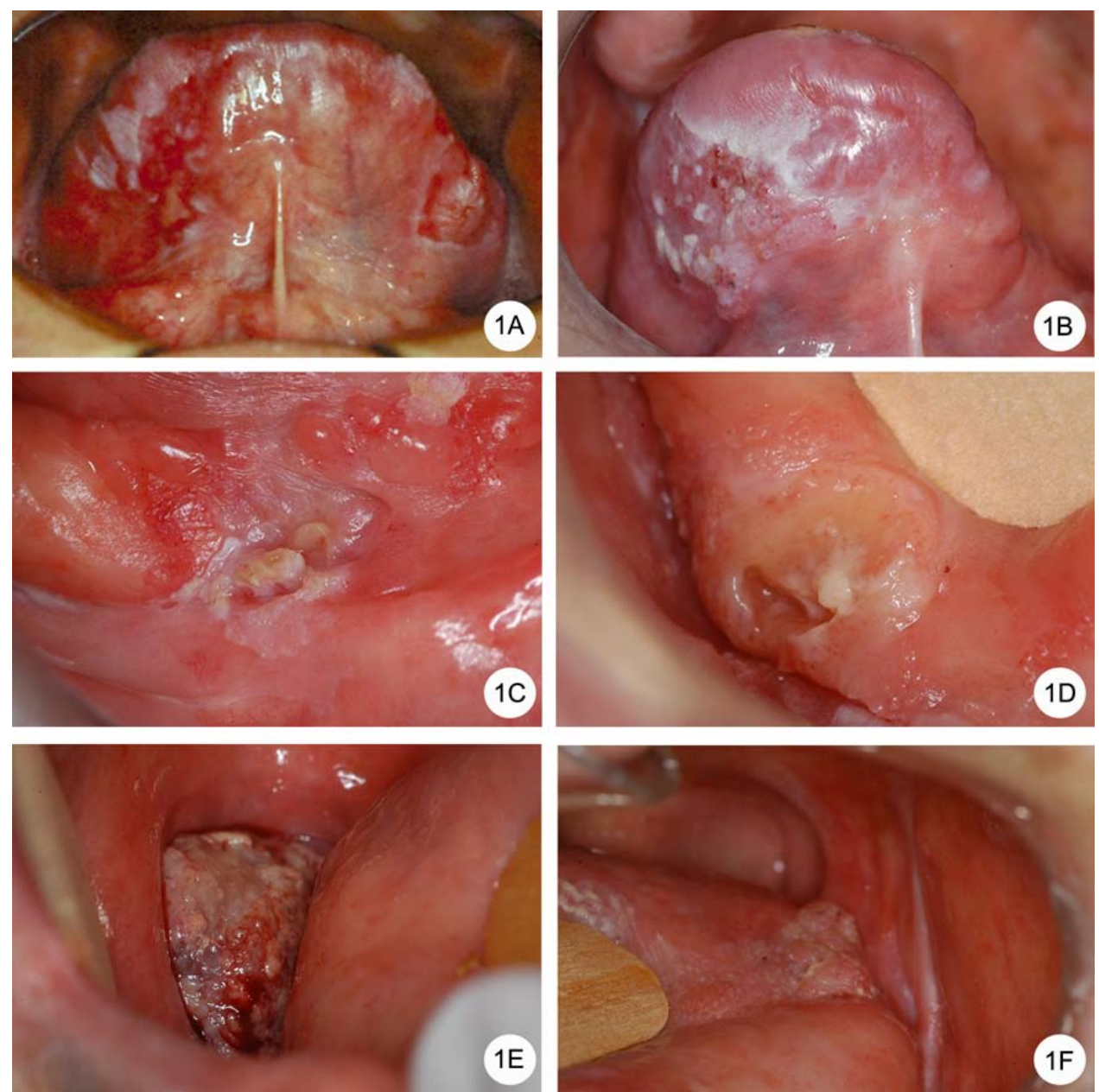

Fig. 1. A.Patient's first clinical presentation: presence of an extensive leukoerythroplastic lesion with some irregular and verrucous areas involving the anterior dorsum and bilateral borders of the tongue.

B. A granular whitish area with ulcerated surface, on the right lateral border of the tongue involving the tip of the tongue and part of the floor of the mouth.

C. Lesion on the right anterior floor of the mouth presenting an irregular keratotic surface, which showed on the histopathological analysis mild epithelial dysplasia.

D. Ulcerated lesion on the right anterior floor of the mouth, diagnosed as squamous cell carcinoma.

E. Necrotic and invasive lesion on the right retromolar area, diagnosed as invasive poorly differentiated SCC.

F. Irregular and granular lesion on the left posterior border of the tongue, which on the histopathological analysis showed severe dysplasia. Later on, a SCC developed at this site. 
alveolar ridge and right inferior alveolar ridge and close periodical evaluation was decided (Table 2). One month later all lesions remained stable but there was an erythroplastic area on the tip of the tongue and a biopsy was performed showing severe epithelial dysplasia (Table 1). All the clinically altered area was removed and the patient was closely followed. However, three months later a whitish lesion with granular surface on the tip and the right lateral border of the tongue developed. A biopsy involving this area was performed and a superficially invasive squamous cell carcinoma was diagnosed (Table 1, Fig. 1B and Fig. 2A). A body screening was performed (abdominal and mammary ultrasound, nasofibrolaryngoscopy and chest X-ray) and, again, no other neoplasia was detected. Thus, a right partial glossectomy was performed. One year and five months after initial presentation, all lesions remained clinically stable with homogeneous surface, however another homogeneous leukoplastic area arose on the right retromolar area. After one year and seven months of follow-up, the right side of the floor of the mouth showed an ulcerated keratotic area. An extensive incisional biopsy was performed and the histopathological analysis detected mild dysplasia (Fig. 1C) (Table 1). Almost eight months after this procedure the same area presented ulceration (Fig. 1D). Another biopsy was performed and a well differentiated invasive SCC was diagnosed, being the patient submitted to another cancer surgery, after a complete body screening (Table 1). In the subsequent consultation, one month later, she presented new leukoplastic lesions on the buccal mucosa bilaterally and verrucous areas on the left inferior alveolar ridge and left posterior border of the tongue. The microscopical analysis of the latter showed severe dysplasia (Fig. 1E). Therefore, she was referred to the surgical oncologist who removed the lesions. After three years of the first clinical evaluation, four months after the former management, the patient detected a $4 \mathrm{~cm}$ palpable lymph node on her right axilla, and then she was seen by her surgical oncologist. Biopsy of the lymph node was performed and the histopathological evaluation revealed a metastatic poorly differentiated SCC (Fig. 2B). A primary breast carci-

Table 1. Summary of the lesions locations and histopathological findings.

\begin{tabular}{|l|l|l|}
\hline \multicolumn{1}{|c|}{ Date } & \multicolumn{1}{c|}{ Biopsied sites } & \multicolumn{1}{c|}{ Histopathological findings } \\
\hline 2002, November & $\begin{array}{l}\text { Dorsum of the tongue and ventral of the tongue } \\
- \text { left and right }\end{array}$ & SCC - poorly differentiated \\
\hline 2003, May & Tongue - tip & Severe epithelial dysplasia \\
\hline 2003, September & $\begin{array}{l}\text { Anterior alveolar ridge and floor of the mouth - } \\
\text { right }\end{array}$ & SCC \\
\hline 2004, June & Floor of the mouth - right & Acanthosis, hyperkeratosis, mild dysplasia \\
\hline 2005, March & Floor of the mouth - right & SCC \\
\hline 2005, August & Lateral posterior border of the tongue - left & Acanthosis, hyperkeratosis, severe dysplasia \\
\hline 2006, January & Floor of the mouth - right & Invasive poorly differentiated SCC \\
\hline 2006, January & Lateral border of the tongue - left & Invasive poorly differentiated SCC \\
\hline
\end{tabular}

Table 2. Summary of the chronological leukoplastic lesions development.

\begin{tabular}{|c|c|c|c|c|c|c|c|c|c|c|c|}
\hline \multirow{4}{*}{ Date } & \multicolumn{11}{|c|}{ Leukoplastic lesions locations } \\
\hline & \multicolumn{4}{|c|}{ Alveolar ridge } & \multicolumn{2}{|c|}{$\begin{array}{l}\text { Buccal } \\
\text { mucosa }\end{array}$} & \multicolumn{3}{|c|}{ Tongue } & \multirow[t]{2}{*}{$\begin{array}{c}\text { Soft } \\
\text { palate }\end{array}$} & \multirow[t]{2}{*}{$\begin{array}{l}\text { Floor of } \\
\text { the mouth }\end{array}$} \\
\hline & Supe & rior & Lov & & & & & & & & \\
\hline & Right & Left & Right & Left & Right & Left & Right & Left & Anterior & & \\
\hline $\begin{array}{l}2002 \\
\text { November }\end{array}$ & & & & & & & $\mathbf{x}$ & $\mathbf{x}$ & $\mathbf{x}$ & & \\
\hline 2003 April & $\mathbf{x}$ & & $\mathbf{x}$ & & $\mathbf{x}$ & & $\mathbf{x}$ & $\mathbf{x}$ & $\mathbf{x}$ & $\mathbf{x}$ & \\
\hline $\begin{array}{l}2003 \\
\text { September }\end{array}$ & & & & & & & $\mathbf{x}$ & & $\mathbf{x}$ & & \\
\hline 2004 April & & & $\mathbf{x}$ & & & & & & & & $\mathbf{x}$ \\
\hline 2005 April & & & & $\mathbf{x}$ & $\mathbf{x}$ & $\mathbf{x}$ & & $\mathbf{x}$ & & & $\mathbf{x}$ \\
\hline
\end{tabular}



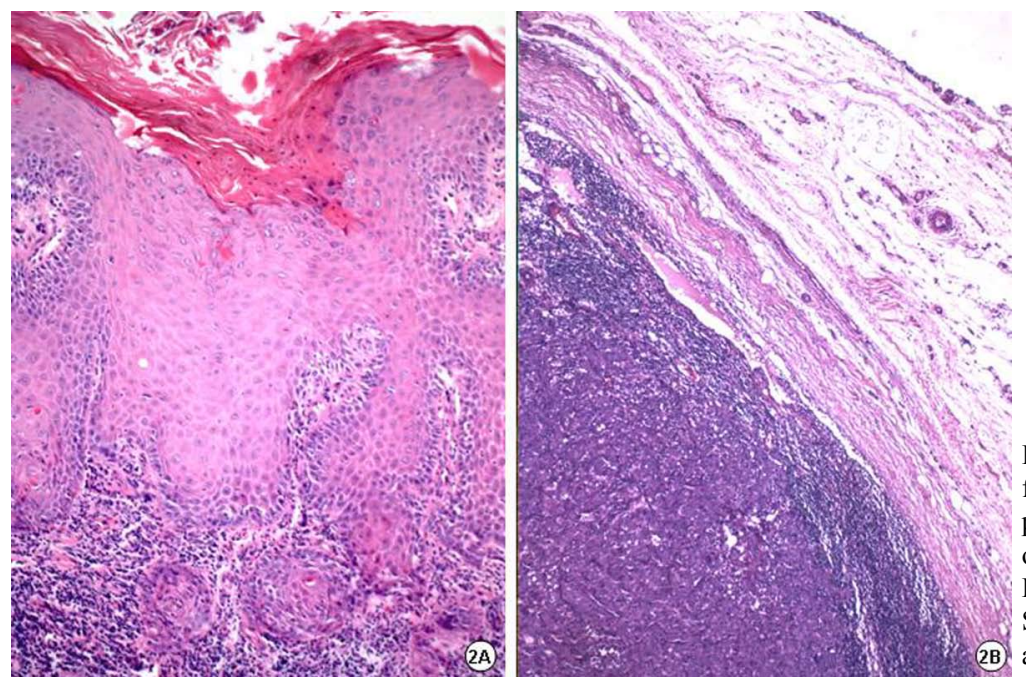

Fig. 2. A. Microscopical aspect of the biopsy performed to the lesion shown on Fig $1 \mathrm{~A}$, showing superficial invasion of SCC (haematoxylin and eosin, original magnification $\times 200$ ).

B. Microscopy of poorly differentiated metastatic SCC in right axillary lymph node (haematoxylin and eosin, original magnification $\times 50$ ).

noma was suspected but there were negative findings on the mammogram. A body screening (abdominal and mammary ultrasound, nasofibrolaryngoscopy and chest $\mathrm{X}$-ray) did not show any tumor. The patient was referred to the Orocentro and an aggressive lesion was identified on the right side involving lateral border of the tongue, floor of the mouth and alveolar ridge and another lesion on the left posterior lateral border of the tongue (Fig. $1 F)$. Biopsies of both areas were performed by her surgical oncologist and the microscopical analysis in both sites showed poorly differentiated invasive SCC (Table 1). Thus, one month later, the tumors were removed and a radical right axillary limphadenectomy was performed. Twenty-four lymph nodes were removed and just one level I lymph node presented metastatic SCC. Adjuvant chemotherapy was initiated (with cisplatin and fluoruracil), but because of the patient's systemic condition just one cycle was performed. The patient developed pulmonary metastases two months after the chemotherapy cycle and died approximately six months after the removal of the tumors.

\section{Discussion}

This very aggressive type of oral leukoplakia presents slow and persistent growth and high rates of malignant transformation (1-8). Batsakis et al. (1999) found SCC development occurrence in almost $100 \%$ of proliferative verrucous leukoplakia, an observation also corroborated by Silverman and Bagan, who found malignant transformation in $87 \%$ and $63,3 \%$ of PVL patients, respectively $(3,4,9)$. Clearly, PVL is resistant to the available treatment modalities, such as cold knife surgery, CO2.laser evaporation, laser surgery, chemotherapy, radiotherapy and presents frequent recurrences (8).

Distant metastases from head and neck SCC are rela- tively common, mainly after recurrence of local or regional disease, but metastases from the upper aerodigestive tract to the axillary lymph nodes is an unusual event $(12,14)$. The metastases drainage way can be suggested mainly by location, size of the primary tumor and regional lymph nodal control (11). Under certain conditions, however, this drainage way may become unpredictable with some metastases bypassing the expected lymph node group ("skip metastases"), occurring at the contralateral side of the neck (cross-over of the lymphatic drainage) or even affecting axillary lymph nodes $(12,13)$. Occurrence of axillary lymph nodes metastases in head and neck carcinoma is a very rare event, varying from $2-9 \%$. Nevertheless, this incidence may be higher because impalpable lymph nodes sometimes are not detected $(15,16)$. The axilla contains numerous lymph nodes, which follow along the axillary venous system and drain the anterolateral chest wall and the upper extremity. Complex and variable connections with lymphatic vessels of the chest and axilla do exist and, under certain conditions, the axillary lymph nodes can become the major lymphatic drainage site from the anterior and lateral neck (12). Changes in the lymphatic drainage can be promoted by a malignant tumor by metastatic blockage of lymph nodes. Consequent fibrosis by surgical manipulation or radiotherapy is also another possible factor that leads to new lymphatic channels formation or aberrant lymphatic channels development (16). Also, there are some other explanations for axillary metastases from oral SCC: hematogenous dissemination; metastases from a second primary tumor along the aerodigestive tract; tumor dissemination after a paraestomal recurrence and retrograde dissemination due to blockage of the jugulo-subclavian junction (15). Our patient had a neck dissection, radiotherapy and re- 
current disease, all possible factors involved in the alteration of the normal lymphatic net drainage that may lead to axillary metastasis.

The poor prognosis in cases with axillary metastasis may result from the high risk of simultaneous occurrence of other distant metastasis (16). Presentation of distant metastasis is usually in the late course of the disease and almost invariably means an unfavorable prognosis (11). Pulmonary metastases are the most frequent in HNSCC, accounting for $66 \%$ of distant metastases. Other sites include bone (hip, long bones or vertebral column), liver, skin, mediastinum and bone marrow (11). Patients with prior history of axillary metastases should have regular monitoring of their lymph nodes as part of their routine follow-up. Frequently palpation and, in suspicious cases, an ultrasound examination or CT should be done (16). Knowledge of this possibility is very important to reinforce the need of early recognition and timely surgically treat axillary metastasis before development of another distant metastasis, improving survival rates.

The present PVL case represented a management challenge, since the leukoplastic lesions presented rapid onset and progression, there was development of multiple SCC and latter on distant metastasis. Even with this impressive course, this case illustrates very well the PVL characteristics previously reported in the literature: resistance to all instituted treatments, frequent recurrences and high rates of malignant transformation. Although axillary metastasis from oral SCC is uncommon, this event was also observed in our patient, emphasizing the importance of close follow-up and careful examination.

\section{References}

1. Hansen LS, Olson JA, Silverman S Jr. Proliferative verrucous leukoplakia. A long-term study of thirty patients. Oral Surg Oral Med Oral Pathol. 1985;60:285-98.

2. Zakrzewska JM, Lopes V, Speight P, Hopper C. Proliferative verrucous leukoplakia: a report of ten cases. Oral Surg Oral Med Oral Pathol Oral Radiol Endod. 1996;82:396-401.

3. Silverman S Jr, Gorsky M. Proliferative verrucous leukoplakia: a follow-up study of 54 cases. Oral Surg Oral Med Oral Pathol Oral Radiol Endod. 1997;84:154-7.

4. Batsakis JG, Suarez P, el-Naggar AK. Proliferative verrucous leukoplakia and its related lesions. Oral Oncol. 1999;35:354-9.

5. Lopes MA, Pazoki AE, Ord RA. Proliferative verrucous leukoplakia: a case report. Gen Dent. 2000;48:708-10.

6. Barnes L, Eveson JW, Reichart P, Sidransky D. World Health Organization Classification of Tumours. Pathology \& Genetics - Head and Neck Tumors. Lyon: IARC Press; 2005. p. 180-81.

7. Cabay RJ, Morton TH Jr, Epstein JB. Proliferative verrucous leukoplakia and its progression to oral carcinoma: a review of the literature. J Oral Pathol Med. 2007;36:255-61.

8. Van der Waal I, Reichart PA. Oral proliferative verrucous leukoplakia revisited. Oral Oncol. 2008;44:719-21.

9. Fettig A, Pogrel MA, Silverman S Jr, Bramanti TE, Da Costa M, Regezi JA. Proliferative verrucous leukoplakia of the gingiva. Oral Surg Oral Med Oral Pathol Oral Radiol Endod. 2000;90:723-30.

10. Bagan JV, Jimenez Y, Sanchis JM, Poveda R, Milian MA, Mu- rillo J, et al. Proliferative verrucous leukoplakia: high incidence of gingival squamous cell carcinoma. J Oral Pathol Med. 2003;32:37982.

11. Morton TH, Cabay RJ, Epstein JB. Proliferative verrucous leukoplakia and its progression to oral carcinoma: report of three cases. $\mathrm{J}$ Oral Pathol Med. 2007;36:315-8.

12. Ferlito A, Shaha AR, Silver CE, Rinaldo A, Mondin V. Incidence and sites of distant metastases from head and neck cancer. ORL J Otorhinolaryngol Relat Spec. 2001;63:202-7.

13. Koch WM. Axillary nodal metastases in head and neck cancer. Head Neck. 1999;21:269-72.

14. Dias FL, Lima RA, Kligerman J, Farias TP, Soares JR, Manfro $\mathrm{G}$, et al. Relevance of skip metastases for squamous cell carcinoma of the oral tongue and the floor of the mouth. Otolaryngol Head Neck Surg. 2006;134:460-5.

15. Rayatt SS, Dancey AL, Fagan J, Srivastava S. Axillary metastases from recurrent oral carcinoma. Br J Oral Maxillofac Surg. 2004;42:264-6.

16. Kowalski LP. Noncervical lymph node metastasis from head and neck cancer. ORL J Otorhinolaryngol Relat Spec. 2001;63:252-5. 